\title{
Développement de Plécoptères (Insecta) dans un ruisseau permanent du Liban'
}

\author{
N.J. Alouf 2
}

Mots clés : Plécoptères, Liban, ruisseaux permanents, cycles biologiques.

Le développement larvaire des Plécoptères Leuctra hippopus Kempny, Siphonoperla libanica Alouf et Protonemura zernyi Aubert est étudié. Ces espèces colonisent des ruisseaux permanents du Liban. L. hippopus est hivernale univoltine ; $S$. libanica est printanière univoltine ; $P$. zernyi a une période de vol étendue de l'automne jusqu'au printemps avec un ralentissement des émergences au début de l'hiver. $\boldsymbol{P}$. zernyi est probablement une espèce plurivoltine.

\section{Development of Plecoptera (Insecta) in a permanent stream in Lebanon}

Keywords : Plecoptera, Lebanon, permanent stream, biological cycle.

A study was made of larval development in the Plecoptera Leuctra hippopus Kempny, Siphonoperla libanica Alouf and Protonemura zernyi Aubert. These species live in a permanent stream in the Lebanon. L. hippopus is a winter, univoltine species; $S$. libanica is a spring, univoltine species, $P$. zernyi has a flight period from autumn to spring with a slackening of emergence rate at the start of winter. $P$. zernyj is probably a plurivoltine species.

Le développement larvaire des Plécoptères au Liban peut être du type semivoltin - Marthamea beraudi - (Alouf 1984) ou univoltin - Capnia? arensi, Protonemura libanocypria, Brachyptera galeata et Isoperla Iibanica (Alouf 1989). L'étude des récoltes de larves et d'adultes de Leuctra hippopus, Siphonoperla libanica et Protonemura zernyi permettent d'approfondir nos connaissances sur les cycles de développement de ces espèces.

\section{Sites et méthodes de récolte}

Ces trois Plécoptères colonisent la rivière Yahfufah de l'Anti Liban, $P$. zernyi se rencontre aussi dans le secteur permanent du ruisseau Qab Ilias du Liban (fig. 1). Les différentes caractéristiques de ces cours d'eau sont données dans des travaux antérieurs (Alouf 1983, 1984, Alouf \& Slim 1984); nous ne rappelons ici qu'une brève description des stations.

1. Travail subventionné par le C.N.R.S. du Liban.

2. Université Libanaise, Faculté des Sciences. Correspondance : Nicolas J. Alouf, Quartier Barbara, Zahlé, Liban.
La rivière Yahfufah est permanente, son lit est creusé dans un massif karstique, sa largeur moyenne est de $8 \mathrm{~m}$ et sa profondeur de $40 \mathrm{~cm}$. La température de l'eau varie de 10 à $15^{\circ} \mathrm{C}$ sans variations thermiques notables entre le jour et la nuit.

La série étudiée provient des récoltes mensuelles effectuées de septembre 1980 à mai 1982 , à cinq stations différentes. Le ruisseau Qab Ilias entre 980 et $1200 \mathrm{~m}$ a été prospecté mensuellement de décembre 1977 à janvier 1979 : la température de l'eau varie de 9 à $15^{\circ} \mathrm{C}$ (fig. 2). Les récoltes larvaires ont été faites avec un filet de Surber de $30 \mathrm{~cm}$ de côté et de $0,3 \mathrm{~mm}$ de vide de maille. Les imagos ont été chassés à vue. La mesure de la largeur de la capsule céphalique sert de repère à l'identification des stades larvaires.

\section{Développement larvaire et période de vol}

\subsection{Leuctra hippopus Kempny}

Cette espèce colonise l'Europe, l'Asie Mineure et la côte levantine (Lillehammer 1986, Bromley 1988). 
La population de Yahfufah est macroptère. La période de vol s'étend sur les quatre premiers mois de l'année : mars-avril en 1974, mi-janvier à mars en 1975 et avril en 1985. Les stades larvaires se rencontrent de septembre à janvier (fig. 3). Les prélèvements de 1974 et 1975 (perdus) signalent la présence de larvules dès les mois de juin-juillet. C'est une espèce univoltine avec une courte période d'incubation des oufs et un développement larvaire automno-hivernal (Lillehammer et al. 1989). La période d'émergence est précoce.

\subsection{Siphonoperla libanica Alouf}

Les stades larvaires sont récoltés de septembre à février (1980-1982). Les adultes ont été récoltés le 10 avril 1985. C'est une espèce probablement printanière précoce à courte période d'incubation des cufs et à développement larvaire automno-hivernal (fig. 4). Nous avons récolté aux printemps 1974 et 1975 des larves dans le cours d'eau côtier Nahr Damour. Les dates de relevés des larves signalées par Berthélemy \& Dia (1982) dans les réseaux côtiers du Liban méridional (sub Pontoperla sp.) concordent avec nos observations.

\subsection{Protonemura zernyi Aubert}

Cette espèce est la plus commune au Livian et déborde sur les régions limitrophes (Por et al. 1985, Bromley 1988). La largeur de la capsule céphalique a servi de repère de croissance pour la population larvaire de Yahfufah et la taille des larves pour celle de Qab Ilias. Berthélemy \& Dia (1982) ayant rencontré cette espèce en automne, hiver et au printemps l'ont définie comme « hivernale et printanière, avec quelques mues imaginales précoces dès l'automne, dans les stations où elles sont abondantes 》. L'analyse de la population larvaire et les périodes d'émergence des populations en notre possession montrent que le développement de cette espèce, quoique cyclique, n'est point annuel. Deux grandes périodes d'émergence sont observées : une cohorte automnale où prédominent les femelles et une cohorte hiverno-printanière avec un sex-ratio $=1$ (Tableau I, fig. 5). Les larves provenant des pontes automnales se développent en hiver et les émergences

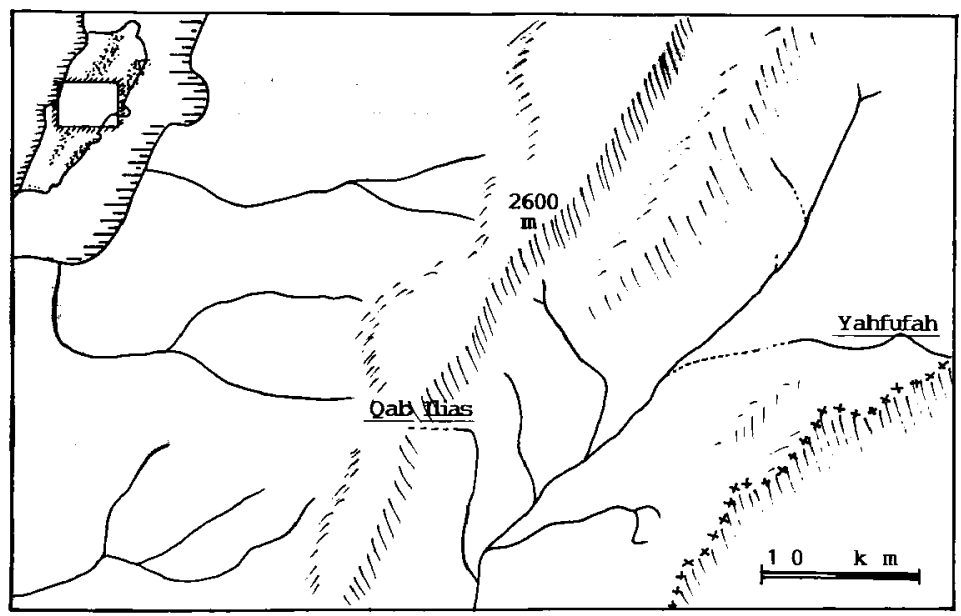

Fig. 1. Carte de la région prospectée. En médaillon, en haut à gauche, le Liban. 

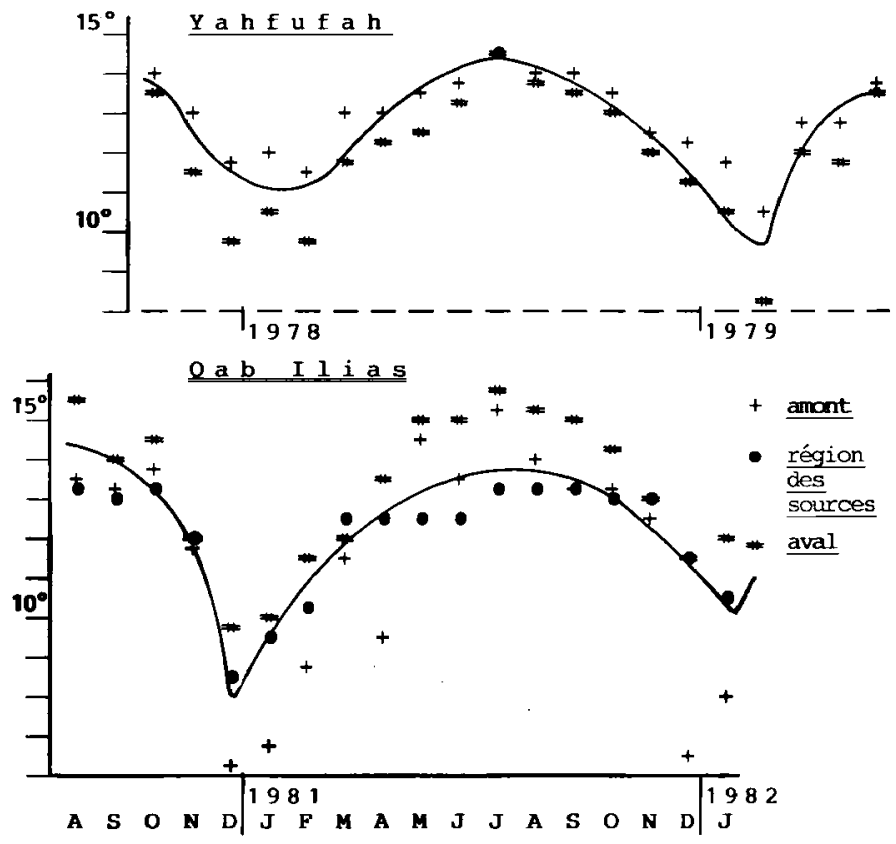

Fig. 2. Evolution de la température, en ${ }^{\circ} \mathrm{C}$, à Yahfufah et à Qab Ilias.

ont lieu au printemps. La nouvelle génération se développe en été et en automne et les imagos émergent en automne et en hiver (fig. 6 et 7 ). On a pensé à la probabilité que dans de bonnes conditions écologiques les émergences automnales débordent sur l'hiver et joignent les vols hivernaux précoces. Les récoltes de l'automne 1990 et celles de la première moitié de l'hiver 1991 où les conditions climatiques étaient particulièrement clémentes (très faible pluviosité et températures douces), infirmèrent cette hypothèse. Les émergences automnales durèrent jusqu'à mi-janvier et les hivernales ne commencèrent que dès la mi-février. Ce hiatus a été observé dans toutes nos récoltes. Bien qu'on ne possède pas de preuves expérimentales, cette espèce ne doit pas souffrir d'une quelconque période de quiescence lui permettant de surmonter la sècheresse estivale et, de ce fait, se trouve confinée au secteur permanent de la rivière Qab Ilias (Alouf 1983). Les relevés de Bromley (1988) sont fragmentaires et n'apportent pas non plus d'éclaircissements sur ce sujet.

\section{Discussion et conclusions}

La sténoécie, et plus particulièrement la sténothermie des secteurs d'altitude des cours d'eau au Liban, consécutives à l'effet régulateur des karsts, permettent 
la succession des générations de $P$. zernyi en moins d'une année. Les pontes hivernale et automnale profitent de l'allongement du jour, la durée de leur croissance sera de 7 à 8 mois. La ponte printanière est à croissance lente et dure de 9 à 10 mois. Cette espèce a trois vols par an provenant de deux lignées qui se chevauchent et décrivent un cycle sur deux ans. La succession est synchronisée avec les saisons : on n'observe que de rares vols au début de l'hiver et aucun vol en été. Peut-on parler de plurivoltinisme? Ou sommes-nous en présence d'une espèce à grande période de vol entrecoupée d'un arrêt ou d'un ralentissement hivernal ? Le cycle biologique est à rapprocher de celui de Nemurella pictetii qui, dans les ruisseaux Breitenbach (Allemagne) et Teichbach (Autriche) a une période de vol qui va d'avril à novembre avec trois pics qui correspondent à trois générations. Cette dernière espèce, à développement
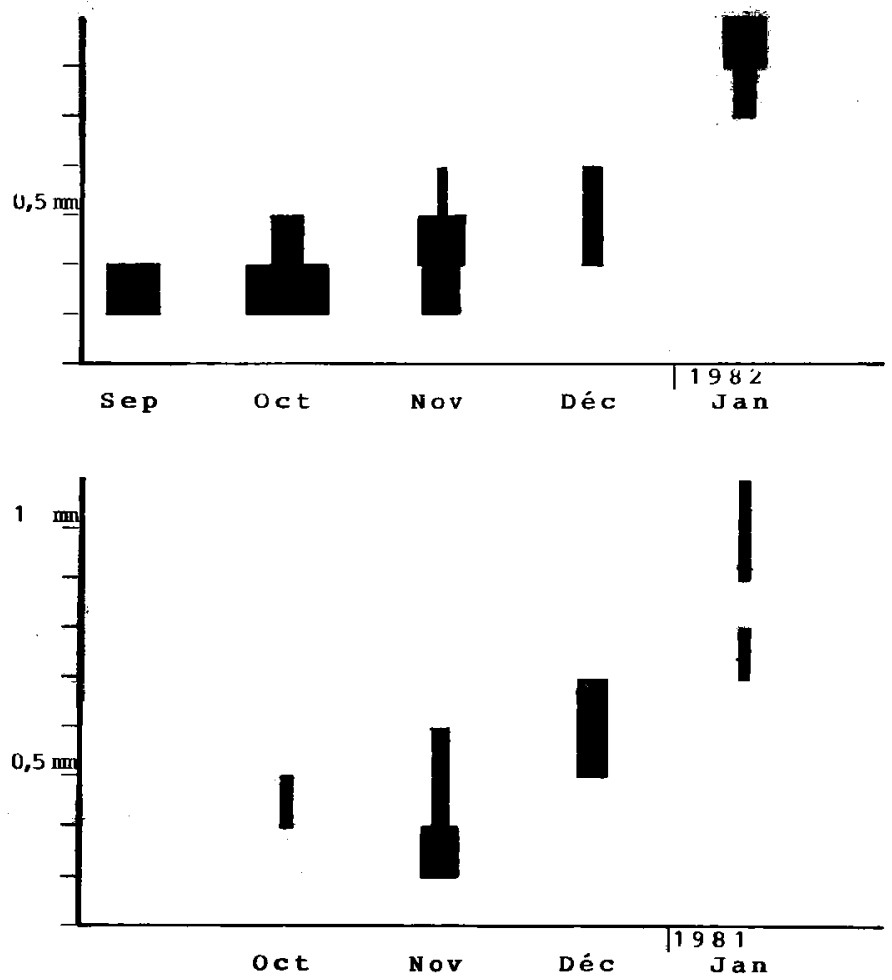

Fig. 3. Leuctra hippopus. Développement larvaire à Yahfufah en 1981 el en 1982. 

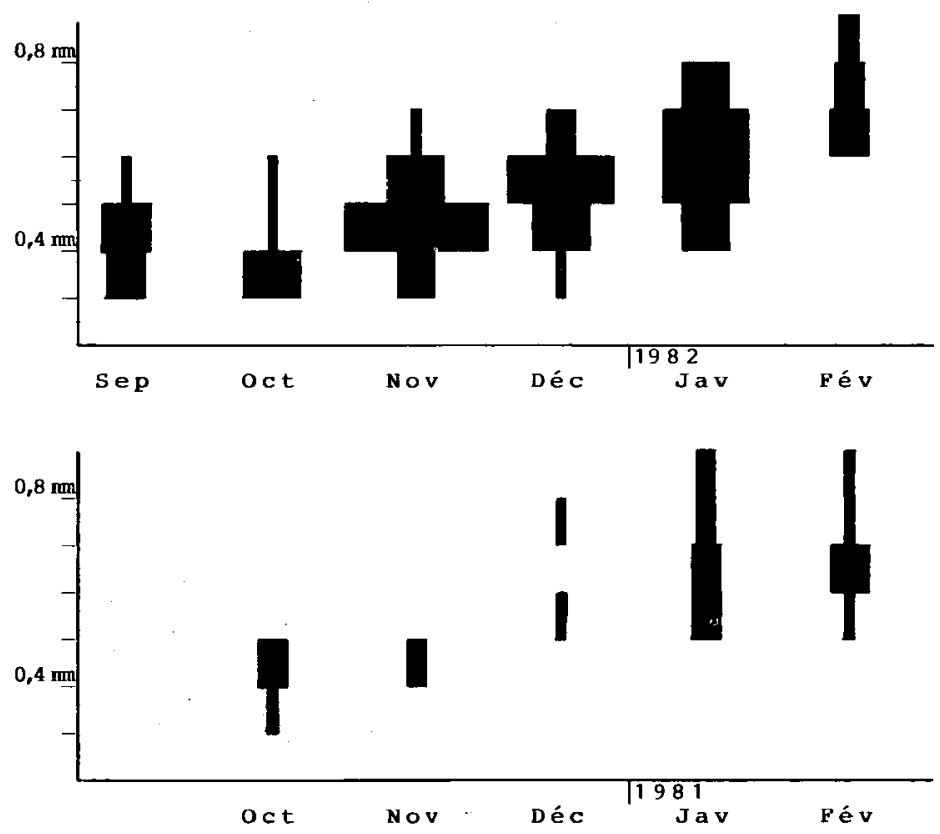

Fig. 4. Siphonoperla libanica. Développement larvaire à Yahfufah en 1981 et en 1982.

direct, achève sa croissance en moins d'une année et serait, selon Wolf \& Zwick (1989), le premier Plécoptère connu à développement plurivoltin.

Le vol des Plécoptères est précoce au Liban. Il est hivernal pour Capnia ? arensi et $L$. hippopus, printanier précoce pour $P$. libanocypria et $B$. galeata, printanier pour $M$. beraudi et $S$. libanica et printanier tardif pour I. libanica (Alouf 1989). L'absence de larvules dans nos récoltes d'été et les mauvaises conditions expérimentales au Liban ne nous ont pas permis de faire des analyses plus rigoureuses et de mesurer les durées effectives d'incubation des œufs et celles de quiescence ou diapause larvaire.
L'éclosion des ceufs doit avoir lieu en été, excepté pour $C$. arensi et $P$. zernyi.

De ce qui précède on peut conclure que le développement des plécoptères au Liban se fait selon les modes suivants :

- Cyclique non annue] : c'est le cas de $M$. beraudi, espece semivoltine et de $P$. zernyi, espèce plurivoltine. C'est le mode « asynchrone " ou « acyclique " de certains auteurs ;

- Cyclique annuel : ou monovoltisme. C'est l'ensemble des autres Plécoptères dont le cycle vital nous est connu. 
Tableau I. Imagos de $P$. zernyi récoltés dans les ruisseaux de Yahfufah et de Qab Ilias.

$\because$ les récoltes de ces deux mois doivent être décalées vers le printemps.

**: voir la figure 5.

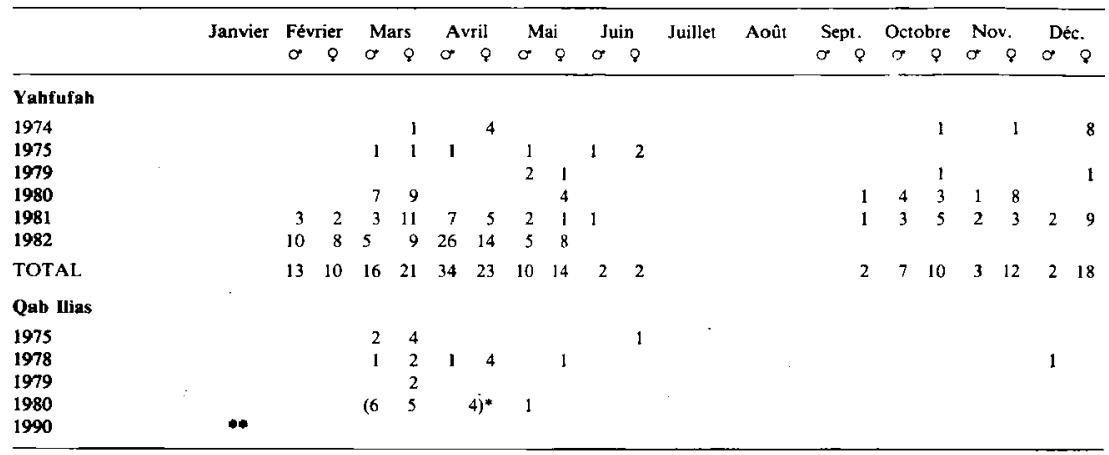

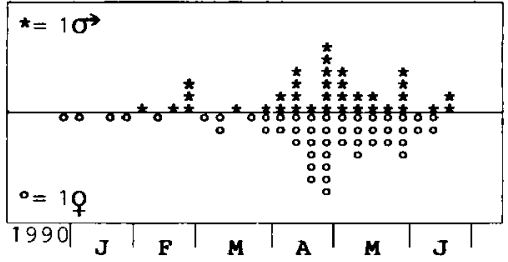

Fig. 5. Imagos de Protonemura zernyi chassés à Qab Ilias du $30 / 12 / 89$ au $26 / 06 / 90$ à raison d'une sortie hebdomadaire. Détail des chasses.

taille en mm

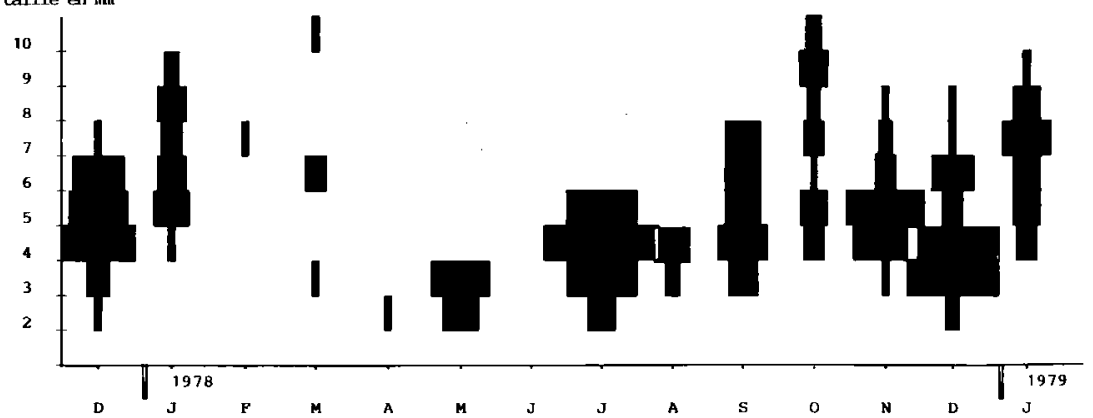

Fig. 6. Protonemura zermyi. Développement larvaire a Qab llias en 1978. 


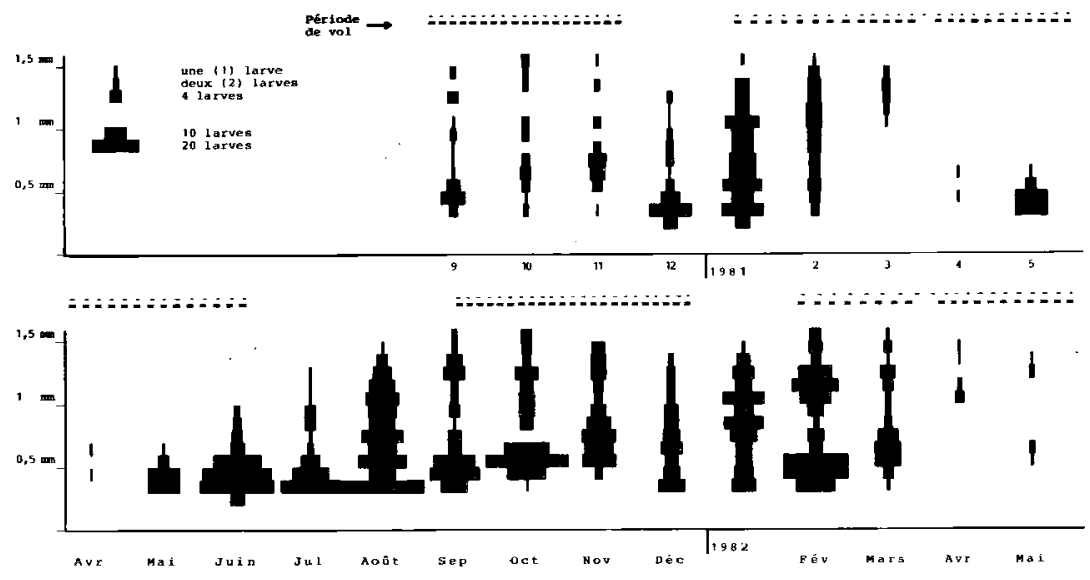

Fig. 7. Protonemura zernyi. Développement larvaire à Yahfufah de septembre 1980 à mai 1982.

\section{Travaux cités}

Alouf (N. J.). 1983. - Contribution à la connaissance de cours d'eau du Liban : la zonation biologique du Nahr Qab Ilias. Annls Limnol., $19:$ 121-127.

Alouf (N.J.). 1984. - Cycle de Marthamea bearaudi Navás dans un cours d'eau du Liban (Plecoptera). Annls Limnol., 20 : $11-16$.

Alouf (N.J.). 1989. - Cycles de vie de quelques Plécoptères (Insecta) d'un ruisseau temporaire du Liban. Annls Limnol., $25: 139-143$.

Alouf (N.J.). 1991 - Description de deux nouveaux Plécoptères (Insecta) du Liban : Capnia bicornata n. sp. et Siphonoperla libanica ก. sp. Senckenbergia Biol.,

Alouf (N.J.) \& Slim (K.). 1984. - Contribution à l'étude de la flore diatomique de Nahr Yahfufah (Liban). Bull. Ins. Sc. Rabat, $8: 95-100$.
Berthélemy (C.) \& Dia (A.). 1982. - Plécoptères du Liban (Insecta). Annls Limnol., i8 : 191-214.

Bromley (H.J.). 1988. - A note on the Plecoptera of Israel. Israel J. Ent. $22: 1-12$.

Lillehammer (A.). 1986. - Taxonomic differences between populations of Leuctra hippopus Kempny (Plecoptera) in Norway. Fatuna Nory. ser. B. $33: 27-32$.

Lillehammer (A.), Brittain (J.E.), Saltveit (S.J.) \& Nielson (P.S.). 1989. - Egg developpement, nymphal growt hand life cycle strategies in Plecoptera. Holartic Ecology, 12 : 173.186.

Por (F.D.), Bromley (H.J.), Dimentman (Ch.), Herbst (G.N.) \& Ortal (R.). 1986. - River Dan, headwater of the Jordan, an aquatic oasis of the Middle East. Hydrobiologia, 134: 121-140.

Wolf (B.) \& Zwick (P.). 1989. - Plurimodal emergence and plurivoltinism of Central European populations of Nemurella pictetii (Plecoptera : Nemouridae). Oecología, $79: 431-438$. 